\title{
Stress distribution within circumferential clasp arms
}

\author{
H. MORRIS, J. W. FARAH, R. G. CRAIG and J. A. A. HOOD \\ Veterans Administration Hospital, Allen Park, Michigan, and University of \\ Michigan School of Dentistry, Ann Arbor
}

\begin{abstract}
Summary
An analysis of stress distributions within circumferential clasp arms with various tapers, was undertaken in an effort to (1) determine the area of maximum stress concentration in both tapered and non-tapered clasps, and (2) to compare this point of maximum stress concentration with the area of frequent clasp breakage. The nontapered clasp showed better distribution of stresses than the tapered clasps. The less favourable stress concentrations could result in eventual failure of the clasp when coupled with other factors such as porosities. The maximum shear stresses in the non-tapered clasp also were lower than those in the tapered clasps. As the taper of the clasp became more accentuated, the magnitude of the stresses increased and shifted towards the tip of the clasp. The maximum tensile stress calculated in a replica of a chromium-cobalt-nickel clasp was in the order of $34 \mathrm{MN} / \mathrm{m}^{2}$ which is well below the ultimate tensile strength of the alloy. This observation leads to the proposal that failure of clasps is a function not only of design but also of other variables, such as porosities, surface irregularities, and fatigue.
\end{abstract}

\section{Introduction}

One of the most frequently used clasp designs in the fabrication of removable partial dentures, is the circumferential clasp. Its function is to prevent the dental prosthesis from being dislodged during mastication. Too often, fracture of the clasp arm occurs during function or adjustment thus creating an inconvenience for both the patient and the dentist. Several reasons for breakage of clasps have been listed by Henderson \& Steffel (1969) among which were the following: (1) repeated flexure in and out of a severe undercut, and (2) breakage from structural failure of the clasp arm. This latter failure could be a result of a nonuniform taper and/or careless finishing and polishing.

Textbooks by Farrell (1971), Dykema, Cunningham \& Johnston (1969) and Applegate (1966), as well as Ney's Surveyor Manual (1968) emphasize the importance of a uniformly tapered clasp arm to prevent a concentration of stresses thereby reducing the fracture of clasps from fatigue failure of the metal. It has been stated (Henderson \& Steffel, 1969) that the dimensions at the tip of a clasp arm should be about half those at the point of attachment for maximum flexibility.

Correspondence: Dr Robert G. Craig, Professor and Chairman, University of Michigan School of Dentistry, Department of Dental Materials, Ann Arbor, Michigan 48109, U.S.A. 


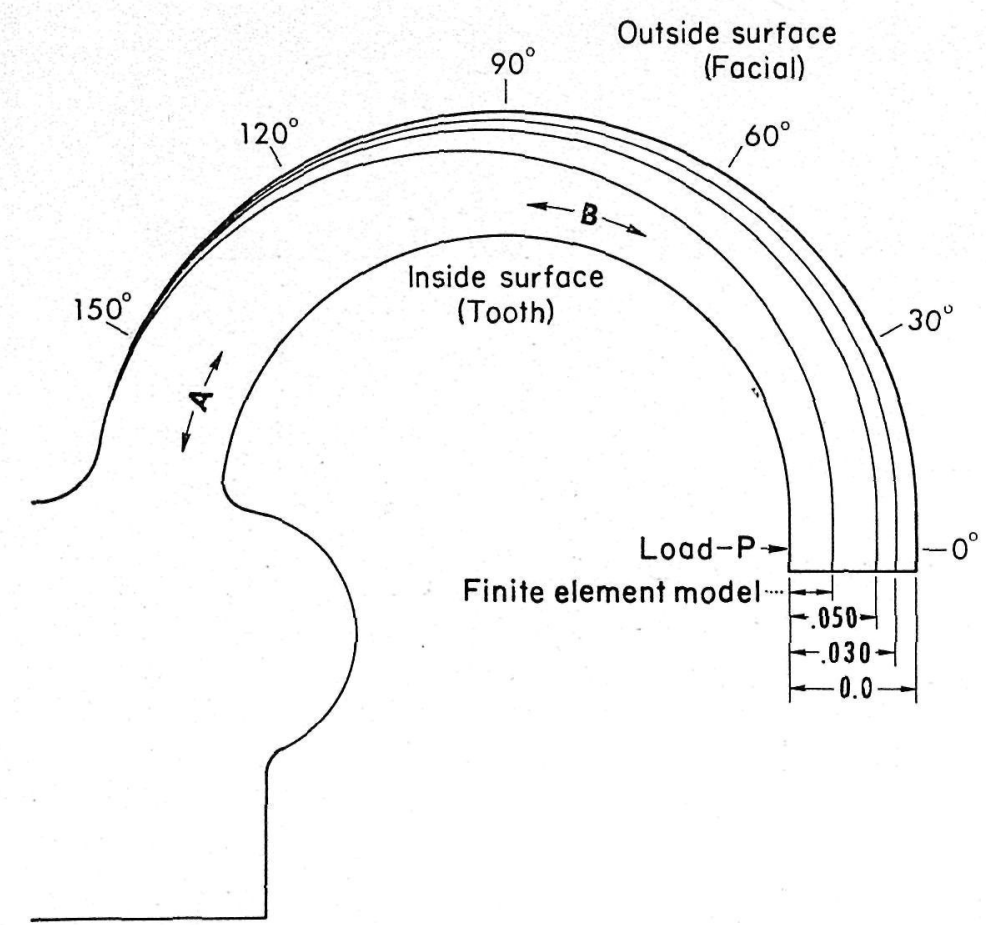

Fig. 1. Schematic of the three photoelastic clasps and the finite element model clasp. A and B indicate areas when the breakage of clasps frequently occur.

Mattila (1964) examined sixty-one removable partial denture frameworks radiographically and found that eighteen exhibited defects within the clasp arm (Fig. 1, area B) and twenty-eight had defects at the junction of the clasp arm with the body of the clasp (Fig. 1, area A).

It has been recently suggested by Dodson \& Peters (1973) that the breakage of clasps during minor adjustments of the completed casting occurred at area B in Fig. 1, while breakage that occurred after prolonged clinical use was at the junction of the clasp arm and body of the prosthesis (Fig. 1, area A).

The purpose of this investigation, therefore, was to determine the region of maximum stress concentration in both tapered and non-tapered models of clasp arms, and to compare the region of maximum stress concentration with the areas of frequent clasp breakage.

\section{Materials and methods}

Twenty-five different straight clasp patterns were purchased and improved stone negatives were prepared. The stone negatives were sectioned at 1, 5, 10 and $15 \mathrm{~mm}$ intervals from the tip, and the thickness of the clasps was determined with a measuring microscope. These measurements were used to calculate the taper for each pattern. The tapers ranged from 0.015 to 0.045 with most clasps exhibiting tapers between $0 \cdot 020$ and $0 \cdot 030$.

Three different methods were used to calculate the stresses and determine the stress distribution in the above clasps: (1) the photoelastic method, (2) the one-dimensional theory of elasticity, and (3) the two-dimensional finite element method.

To prepare the models for the first method, three brass templates were machined into the following tapers: (1) a non-tapered clasp (0.000), (2) a moderately tapered clasp (0.030) and (3) an extremely tapered clasp (0.050) (Fig. 1). Tracings of these templates were made on sheets of photoelastic material* which was then cut to * Photolastic, Inc., Malvern, Pa. 


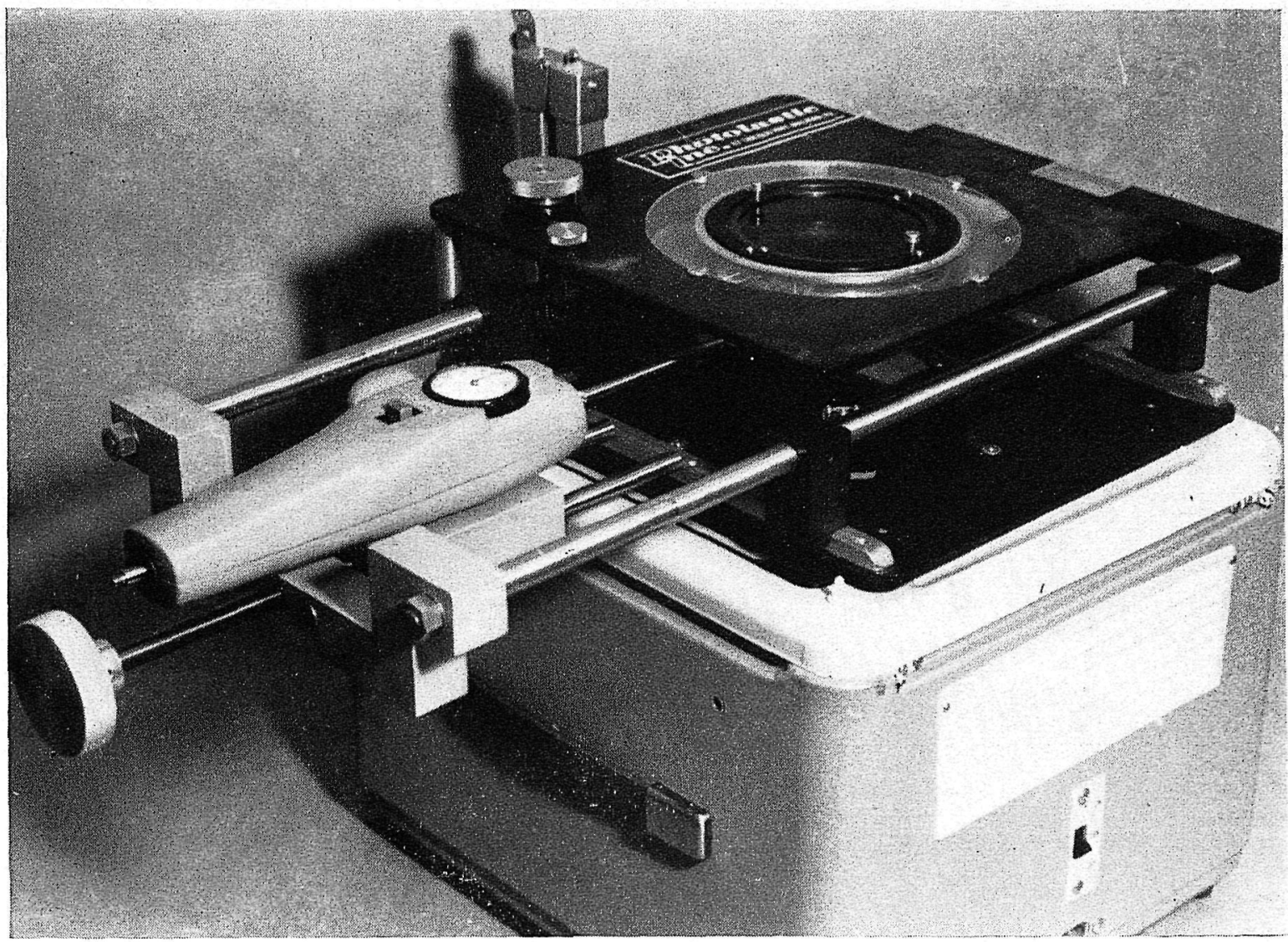

Fig. 2. Modified desk-type photoelastic bench with loading frame.

the desired taper. The photoelastic models were mounted on a modified desk-type photoelastic bench as shown in Fig. 2. The photoelastic clasps located between the analyzer and the polarizer of the polariscope, were loaded in tension from 4.4 to $17 \cdot 6 \mathrm{~N}$ at approximately $2 \cdot 2 \mathrm{~N}$ intervals. Typical isochromatic fringe patterns for the three clasps are shown in Fig. 3.

In the second method, a one-dimensional theory of elasticity approach was used to determine the maximum shear stresses at various points along the clasp. The theoretical value of the maximum shear stress, $\tau_{\max }$, was obtained by using the following equation:

$$
\tau_{\max }=\frac{1}{2} \frac{\mathrm{P} \sin \theta}{\mathrm{A}}+\frac{\mathrm{Mc}}{\mathrm{I}}
$$

where $\mathbf{P}$ is the axial load, $\theta$ is the arbitrary value given to the angle in Fig. 1, $\mathrm{A}$ is the cross-sectional area, $I$ is the moment of inertia, $M$ is the moment at a specified point, and $c$ is one-half the width of the clasp. The values of the theoretical shear stress were plotted and compared to the photoelastic shear stress.

The two-dimensional finite element method was used to determine the stresses at various points within a non-tapered and an arbitrarily tapered clasp (Fig. 1). The finite element method is a numerical analysis method, and its use in dental research was discussed by Farah (1972).

\section{Results}

In Fig. 1, the three clasp designs $(0.000,0.030,0.050$ tapers $)$ are shown and the points at which the fringe order $(\mathrm{N})$ was recorded are indicated. The fringe order, $\mathrm{N}$, which is directly proportional to the maximum shear stress was plotted as a function of the 


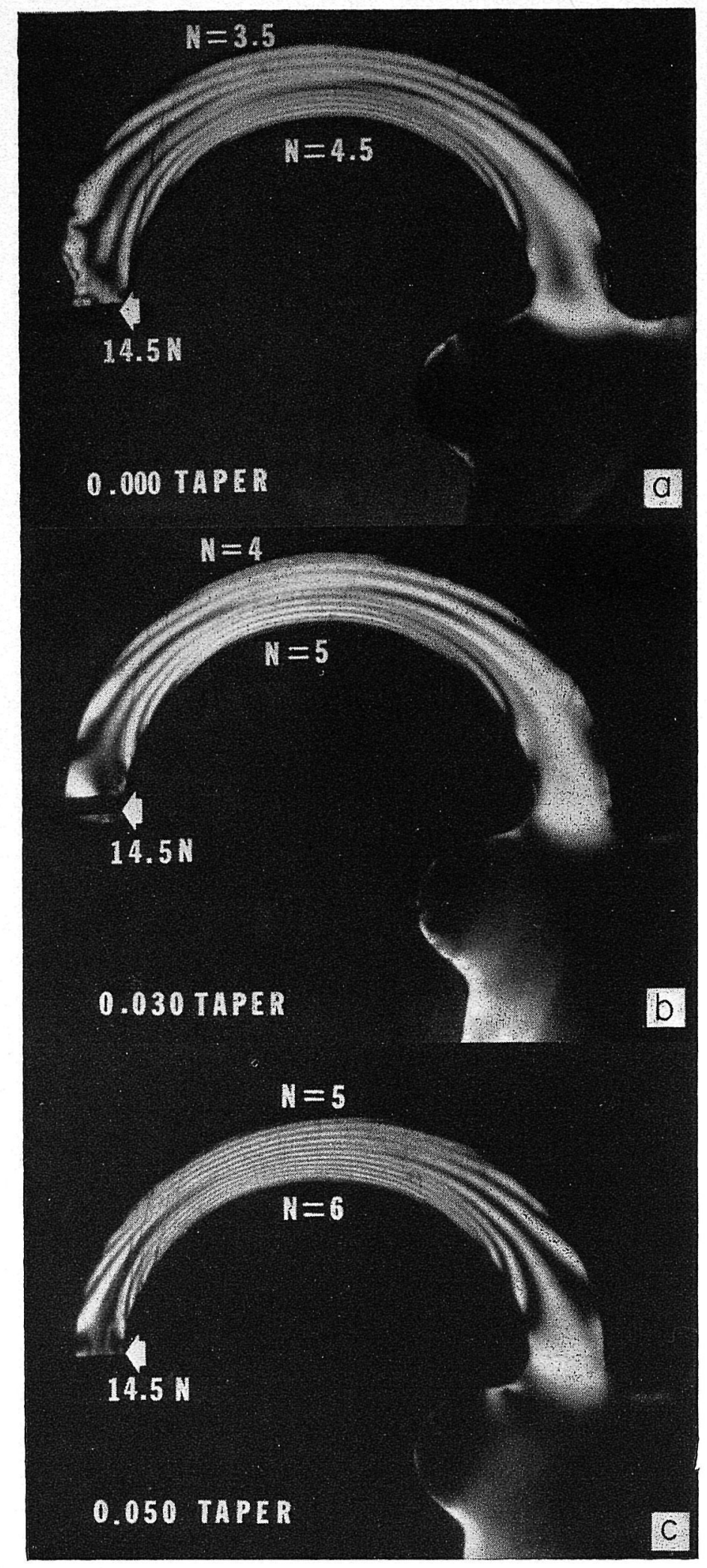

Fig. 3. Typical isochromatic fringe patterns for the three photoelastic clasps. (a) 0.000 taper; (b) 0.030 taper; (c) 0.050 taper.

angle shown in Fig. $1\left(0^{\circ}, 30^{\circ}, 60^{\circ}, 120^{\circ}, 150^{\circ}\right)$. The relationship between $\mathrm{N}$ and the maximum shear stress is given by the equation $\tau_{\max }=\mathrm{Nf} / 2 \mathrm{~h}$, where $\tau_{\max }$ is the maximum shear stress, $\mathrm{f}$ is the material constant (usually provided by the supplier of the photoelastic material), and $\mathrm{h}$ is the thickness of the photoelastic sheet. Also shown in Fig. 1 are the two areas denoted by A and B which indicate the location of frequent clasp breakage as mentioned previously. It should be noted that in all cases, the graphs plotted are a function of $\mathrm{N}$ as measured on the inside of the clasp. This was done 
because the inside of the clasp, under the loading conditions chosen, was in tension and thus more prone to failure than the outside which was in compression. The trends in the stress distribution, however, were the same whether taken on the inside or outside of the clasp.

The photoelastic clasps under a $14.5 \mathrm{~N}$ tensile load are shown in Fig. 3. It can be seen how the stress distribution is affected by the taper of the clasp and how under the same loading conditions for different clasps the fringe order, $\mathrm{N}$, increased from 4.4 in the untapered clasp, to 4.9 in the 0.030 taper and 5.9 in the 0.050 taper.

A plot of the maximum fringe order on the inside of the clasps as a function of the angle at which it was taken is presented in Fig. 4. These curves were obtained with a $4.9 \mathrm{~N}$ load. When the load was doubled to $9.8 \mathrm{~N}$, the curves shown in Fig. 4 shifted

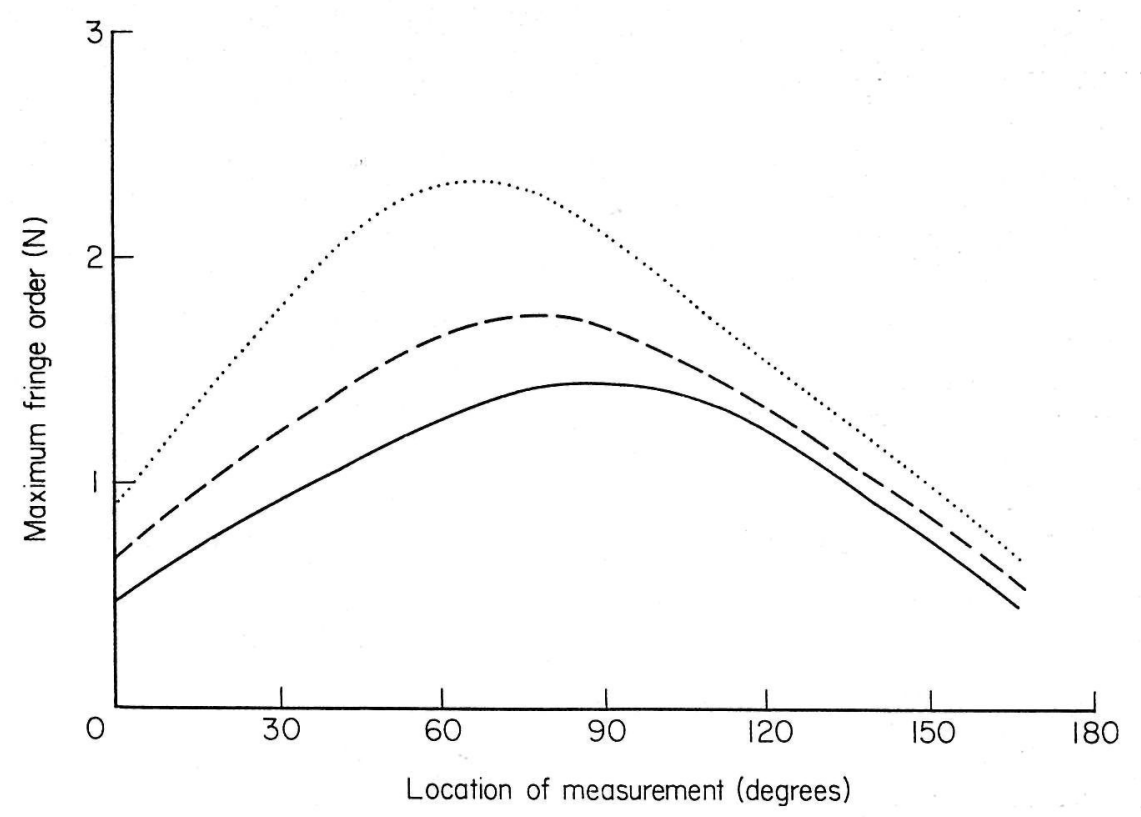

Fig. 4. Distribution of fringe order on the inside surface of the three photoelastic claps with loads of 4.9 N. Taper..... 0.050; 0.030 ; 0.000 .

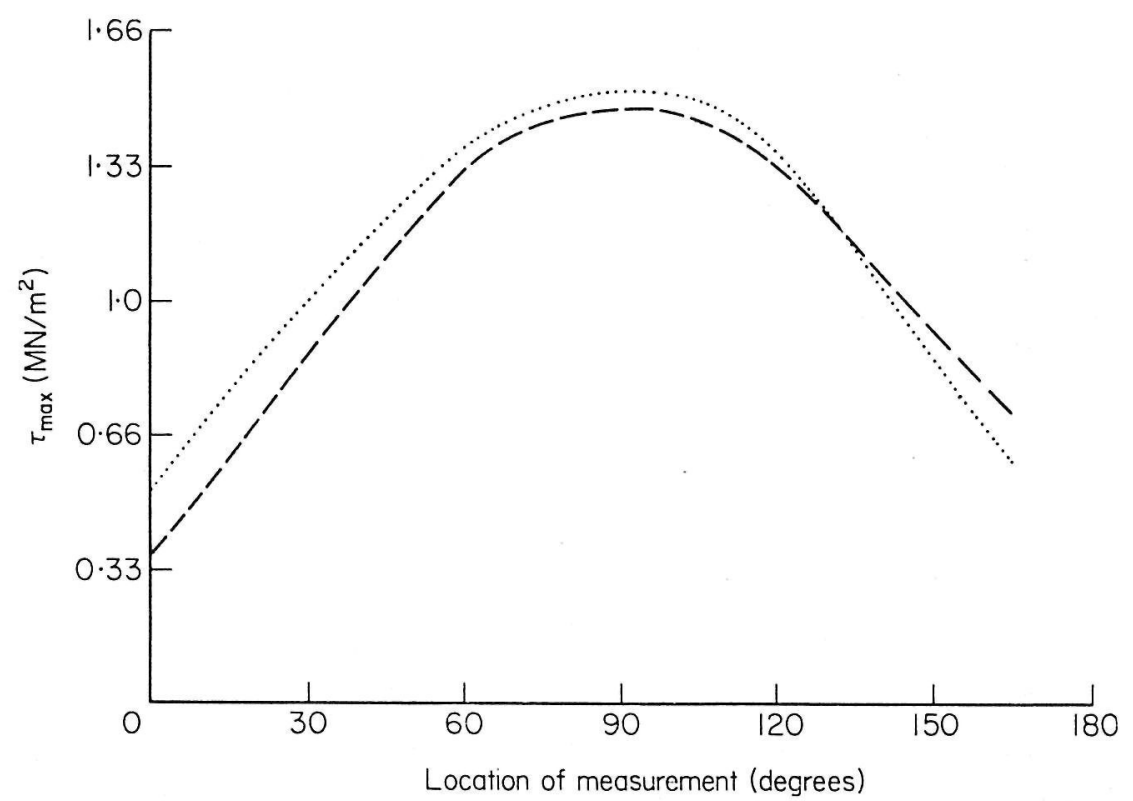

Fig. 5. Comparison of the maximum shear stresses for a non-tapered clasp obtained theoretically $(----)$ and photoelastically $(\ldots \ldots \ldots)$. 


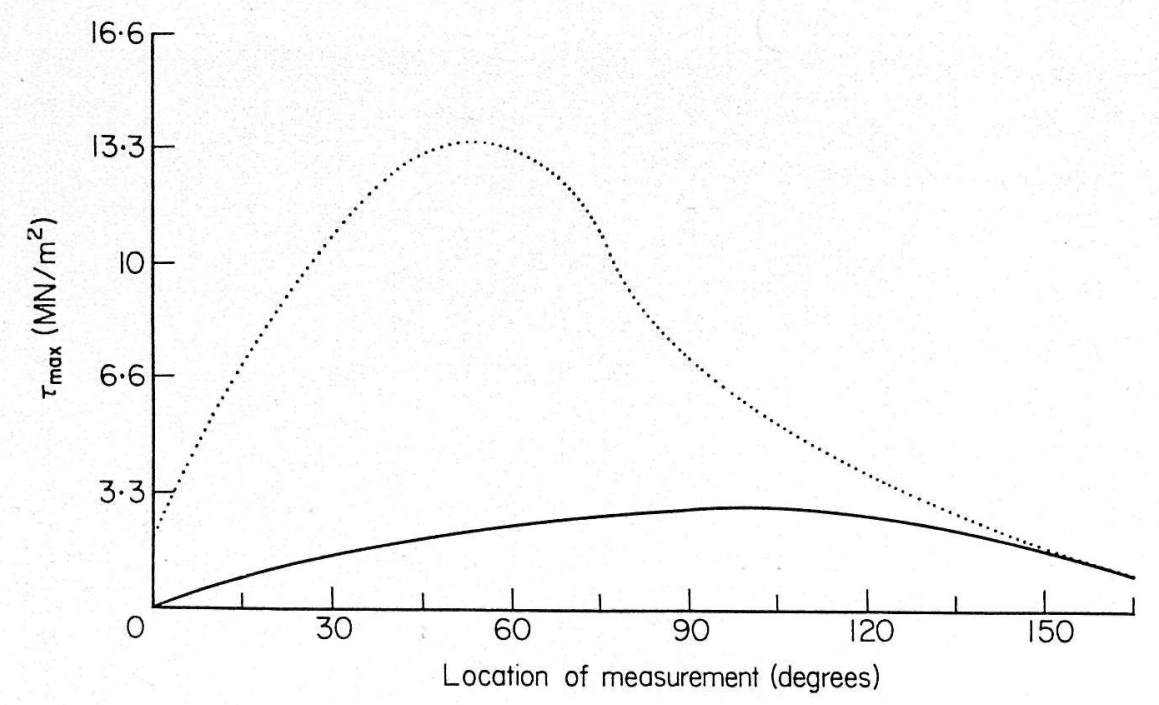

Fig. 6. Surface stresses on a chromium-cobalt alloy (Vitallium) clasp calculated by the finite element method. ........ Tapered; non-tapered.

vertically to a higher level, while maintaining the same trends provided the elastic limit of the material was not exceeded.

In Fig. 5 the maximum shear stresses for a non-tapered clasp obtained photoelastically are compared to the maximum shear stress obtained by a one-dimensional derivation of the theory of elasticity (Equation 1) and reasonable agreement was obtained.

Since the stresses for the tapered clasps are not obtained as readily from the theory of elasticity, a two-dimensional finite element programme was used to calculate the stresses for an arbitrarily tapered and a non-tapered clasp (Fig. 1) and the results are plotted in Fig. 6. In the finite element model the two clasps, representing an actual size chromium-cobalt clasp with a modulus of $21 \times 10^{4} \mathrm{MN} / \mathrm{m}^{2}$ and a Poisson's ratio of 0.33 , were subjected to a tensile load of $12 \cdot 2 \mathrm{~N}$ and the maximum shear stresses were calculated. These stresses were also compared to those obtained by the theoretical and photoelastic methods.

\section{Discussion}

While the breakage of clasps does not constitute a major problem in dentistry, it is nevertheless a situation that both patients and the dentists would prefer to avoid. For years it was believed that the primary cause of the breakage of clasps was the undesirable concentration of stresses within the clasp arm, which resulted in eventual fatigue failure of the alloy. Based on well established engineering principles, it seemed logical to assume that a tapered clasp arm would eliminate this problem. Although numerous clasp patterns and tapers have been made available to the profession, clasps continue to fail. While casting defects have long been recognized as a cause of the failure of clasps, Mattila's work (1964) was the first to give an indication of the magnitude of the problem. He concluded that $10 \%$ of the clasp areas had serious casting defects, and $45 \%$ of the clasps had defects at the junction of the bars and arms, all of which could lead to failure.

The design of the clasp arm should be such that during function favourable stress distributions are present, thus preventing clasp failure due to stress concentrations which would (1) exceed the yield strength of the clasp material resulting in permanent deformation of the clasp arm and (2) provide a point where eventual fatigue failure 


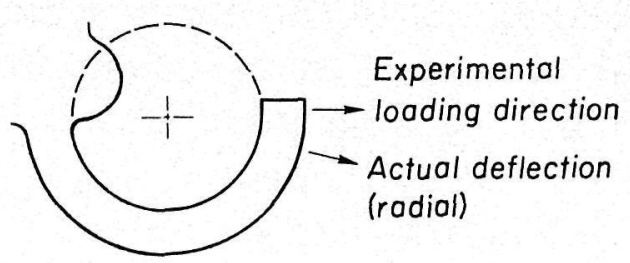

Fig. 7. Direction of loading and deflection in a circumferential clasp.

could result. The model in Ney's photoelastic study (1968) was loaded in such a manner that the clasp arm behaved as a cantilever beam. This resulted in high stress concentrations in the area designated A in Fig. 1.

The mode of loading the models in this study is felt to be more representative of actual clasps in function for the following reason. In clinical use the clasp is usually designed so that the terminal third lies below the survey line. This portion of the clasp arm is subjected to deflections, in the radial direction (Fig. 7) during insertion and removal. The remaining two-thirds, which lies above the survey line, provides lateral stability.

Of the photoelastic models examined, the non-tapered clasp provided the most favourable distribution of stress (Fig. 4). Because of the relatively small difference in tapers of the clasp, little difference in stress distribution could be expected, which is demonstrated in Fig. 4. Fig. 4 also shows how the fringe order $(\mathrm{N})$ varied from the tip of the clasp to the body of the clasp. The maximum value for $\mathrm{N}$ occurred at $90^{\circ}$ for the non-tapered clasp $(0 \cdot 000)$, at $80^{\circ}$ for the moderately tapered clasp $(0 \cdot 030)$, and at $70^{\circ}$ for the clasp with a maximum taper $(0 \cdot 050)$.

The area where $\mathrm{N}$ reached its maximum value, i.e. the span between $70^{\circ}$ and $90^{\circ}$ in Fig. 4, corresponded with area B in Fig. 1. This is the point where the breakage of clasps frequently occurs during minor adjustments at the time of completion of the partial denture framework (Dodson \& Peters, 1973). On the other hand area A is the point of frequent failure after the prosthesis has been in clinical use (Dodson \& Peters, 1973). The present study, however, did not show A to be an area of high stress concentration. Another explanation for the failure of clasps at A and B could be the result of porosities in these areas.

The breakage of clasps in area A could also be the result of torsion of the clasp arm. Torsion would occur under several conditions: (1) during insertion and removal of the prosthesis as the leading edge of the cast clasp arm, half-round in cross-section, comes into contact with the supra-bulge of the abutment tooth, and (2) during mastication. However, it is doubtful that the torquing effect produced during mastication would be of sufficient magnitude to cause clasp breakage. A round retentive clasp arm would eliminate the problem of torsion, for it is the only cross-sectional form that has the same flexibility in all directions. Applegate (1966), as well as numerous other clinicians, favours the use of a retentive clasp arm consisting of a round wrought wire of either a precious or base-metal composition which is attached to the body of the clasp during the casting process, or by soldering after the casting has been completed. He suggested that fracture of this type of retentive clasp arm is possibly a result of recrystallization of the wrought structure, i.e. poor technical procedure. He suggests that the wrought clasp arm to prevent 'concentration of flexures' at the junction of the clasp body and the retentive arm. The data obtained in Fig. 4, however, indicates that the non-tapered clasp exhibits the most favourable distribution of stresses. 
In order to establish the validity of the photoelastic model, the maximum shear stress obtained was compared in Fig. 5 to that obtained theoretically using Equation 1. The maximum shear stresses in the photoelastic non-tapered clasp model were 1.5 $\mathrm{MN} / \mathrm{m}^{2}$, which corresponded closely to those obtained theoretically. The $1.5 \mathrm{MN} / \mathrm{m}^{2}$ shear stress does not reflect the magnitude of the stresses occurring in an actual clasp, since the photoelastic model was considered to be approximately six times the actual size of a clasp arm.

From the results obtained by the finite element method it can be seen that the shear stresses in Fig. 6 for the clasps follow the same trends as observed earlier in Fig. 4 for the photoelastic clasp models. The non-tapered clasp reached a maximum shear stress of approximately $3.1 \mathrm{MN} / \mathrm{m}^{2}$ between $90^{\circ}$ and $100^{\circ}$, which is in agreement with the location of the maximum shear stresses obtained earlier. For the tapered model, the maximum shear stress reached a value of $14 \mathrm{MN} / \mathrm{m}^{2}$ which was more than four times the stress found in the untapered model. The maximum tensile stresses for both the non-tapered and tapered clasps occurred between $90^{\circ}$ and $100^{\circ}$ and were 8.3 $\mathrm{MN} / \mathrm{m}^{2}$ and $34 \mathrm{MN} / \mathrm{m}^{2}$, respectively. When these values are considered in relation to the known ultimate strength of a chromium-nickel base partial denture alloy $(\sim 800$ $\mathrm{MN} / \mathrm{m}^{2}$ ) it is obvious that the loading conditions considered here will not cause immediate clasp failure. However, it seems likely that tapered clasps would be more prone to eventual fatigue failure after prolonged clinical function than non-tapered clasps. Any factor that would enhance the development of an area of stress concentration such as porosities, surface irregularities, investment inclusions, coupled with variations in grain size could lead to failure under normal loading.

\section{References}

APPLEGATE, O.C. (1966) Essentials of removable partial denture prosthesis, 3rd edn. W.B. Saunders, Philadelphia.

Dodson, C.A. \& Peters, R.B. (1973) Personal communications.

Dykema, R.W., Cunningham, D.M. \& Johnston, J.F. (1969) Modern practice in removable partial prosthodontics, 1 st edn. W.B. Saunders, Philadelphia.

FARAH, J.W. (1972) Stress analysis of first molars with full-crown preparations by three-dimensional photoelasticity and the finite element method. Ph.D. dissertation, University of Michigan.

Farrell, J.H. (1971) Partial denture designing: a guide for use in the dental surgery and laboratory, 2nd edn, p. 58. Henry Kimpton, London.

HeNDERSON, D. \& STEFFEL, V.L. (1969) McCracken's partial denture construction, 3rd edn, pp. 132-133, 432-433. C.V. Mosby, St Louis.

Mattila, K. (1964) A roentgenological study of internal defects in chrome-cobalt implants and partial dentures, Acta odontologica Scandinavica, 22, 215.

Ney Co., J.M. (1968) Ney Surveyor Manual, pp. 64-67. J.M. Ney Co., Bloomfield.

Manuscript accepted 30 September 1975 
This document is a scanned copy of a printed document. No warranty is given about the accuracy of the copy. Users should refer to the original published version of the material. 